\title{
ATENDIMENTO DE SAÚDE À CRIANÇA EM SITUAÇÃO DE EXCLUSÃO SOCIAL
}

HEALTH CARE TO CHILDREN IN SITUATION OF SOCIAL EXCLUSION

*Moneda Oliveira Ribeiro, **Karen Murakami Yano, ***Washington Luiz Pereira de Sousa

\section{RESUMO}

O projeto é destinado a atender crianças e adolescentes em situação de risco que frequentam uma entidade não governamental e sem fins lucrativos. São crianças moradoras de habitações coletivas precárias, em bairro central da cidade de São Paulo, com grande potencial para se tornarem moradores de rua. O projeto visa capacitar o estudante de enfermagem a organizar o serviço de saúde e prestar assistência à criança em situação de exclusão social. Sob supervisão docente, o aluno avalia o crescimento e desenvolvimento das crianças, sua história pessoal, familiar e condição habitacional; elabora prescrição, intervenção e evolução de enfermagem; desenvolve orientações de saúde às crianças e familiares; e faz encaminhamentos aos serviços de saúde parceiros. Os estudantes elaboraram os instrumentos de coleta de dados, identificaram as necessidades e problemas de saúde e adotaram intervenções compatíveis com as peculiaridades da criança. A evolução do trabalho mostrou que o projeto é viável, demanda baixo custo e tem potencial para ser replicado com outros grupos sociais.

Palavras-chave: Criança. Exclusão social. Violência.

\section{ABSTRACT}

The project is designed to assist children and adolescents at risk who attend a non-governmental, non-profitable organization. These are children living in precarious tenements in the central district of Sao Paulo, with great chances of becoming homeless. The project aims to enable nursing students to organize health services and assist the child in situations of social exclusion. Under teacher supervision, the student evaluates the growth and development of the children, their personal history, family and living condition; prepare prescription, intervention and outcome of nursing, develops health guidelines for children and families, and makes referrals to health care partners. Students developed the instruments for data collection, identified the needs and health problems and adopted measures consistent with the peculiarities of the child. The evolution of the study showed that the project is viable, demands low cost and has potential to be replicated with other social groups.

Key words: Children. Social exclusion. Violence.

\footnotetext{
* Enfermeira, Professora Doutora do Departamento Enfermagem Materno-Infantil e Psiquiátrica da Escola de Enfermagem da Universidade de São Paulo - e-mail: moneda@usp.br. ** Enfermeira, Especialista em Enfermagem Psiquiátrica e de Saúde Mental, Mestre em Enfermagem Pediátrica. **** Enfermeiro Visitador da ProAtiva Promoção da Saúde.
} 


\section{INTRODUÇÃO}

A exclusão social é fruto da discriminação de uma categoria social de determinado contexto histórico e delimitação geográfica. Ela decorre de eventos políticos, econômicos e culturais de uma ordem social. No Brasil, está associada principalmente à pobreza. Tal condição priva as famílias carentes do acesso a bens e serviços essenciais (educação, saúde, alimentação, trabalho, moradia, lazer, segurança, previdência social), conforme especificado na Constituição Federal de I988. Essas famílias constituem grupos sociais em situação de risco e/ou vulnerabilidade.

As crianças são as mais prejudicadas quando se considera sua condição de estar em desenvolvimento. Restritas em usufruir seus direitos sociais, ficam mais suscetíveis a enfermidades, estando em risco seu pleno desenvolvimento ou mesmo sua sobrevivência. A exclusão social as torna vulneráveis à violência (física, psíquica e social) à medida que buscam suprir suas necessidades com trabalho informal em locais insalubres ou com envolvimento em atividades ilícitas.

A exclusão social constitui uma violência social, dada a condição de exposição às situações de risco. $\mathrm{O}$ número de homicídios em 2001 envolvendo crianças e adolescentes (entre O e I 8 anos) chegou a 5.92I mortes por IOO mil habitantes, o equivalente a I2,89\% do total de homicídios ocorridos no ano [3].

Em 200I, havia no Brasil 36.313.345 crianças de 5 a I5 anos (29.470.985 do meio urbano e 6.842.360 do meio rural). Destas, 3.094.249 trabalhavam (correspondente a $8,5 \%$ do total), mas somente $\mathrm{I} .097 .776$ crianças recebiam pagamento $(35,5 \%$ das que trabalhavam). Na zona urbana, a maioria das crianças que trabalhava recebia pagamento $(56,2 \%$ dos meninos e $62,4 \%$ das meninas), mas na zona rural somente $12,7 \%$ dos meninos e $\mathrm{I} 4,8 \%$ das meninas que trabalhavam recebiam pagamento. Tomando como referência a população de criança entre 7 e I 5 anos, $86,3 \%$ delas só estudava, as demais ( $13,7 \%)$ estudava e trabalhava, ou só trabalhava, ou não estudava nem trabalhava [3].

A violência sempre foi concebida como um problema social, mas muitos problemas de saúde decorrem de problemas sociais. Devido aos consideráveis índices crescentes de morbidade e mortalidade decorrentes da violência, esta deixou de se restringir a um fenômeno social e passou a ser reconhecida como um agravante de saúde. Somente a partir da décima edição da Classificação Internacional de Doenças (CID) da Organização Mundial da Saúde (OMS), a violência foi incluída no item "Causas Externas", vindo a constituir um problema de saúde pública [1].

A assistência de saúde à criança em situação de exclusão e/ou violência consiste em identificar e avaliar as demandas mais urgentes e estabelecer prioridades, organizadas em três etapas: avaliar a necessidade de indivíduos/grupos de risco; estabelecer prioridades de saúde; elaborar intervenções para promoção, proteção ou recuperação da saúde [2].

A violência contra a criança costuma ser discreta, sem evidências claras de agressões. A equipe de saúde precisa estar atenta aos sinais de violência e abordar membros da família e da comunidade para identificar situações de risco.

Alguns setores sociais têm se mobilizado para organizar redes de atendimento à criança vítima de violência e para capacitar os profissionais de saúde a identificar sinais e evidências de violência contra a criança. Mas ainda é muito pouco para se obter resultados efetivos. Entre as alternativas pertinentes, é necessário inserir nos currículos de graduação o ensino sobre como lidar com essa problemática.

$\mathrm{Na}$ Escola de Enfermagem da Universidade de São Paulo, há algumas iniciativas nessa direção. O projeto de extensão à comunidade intitulado "Atendimento de saúde à criança em situação de exclusão social”, vinculado ao grupo de pesquisa do CNPq intitulado "A criança em situação de exclusão social e/ou violência", provê estratégias de ensino com o intuito de despertar sentimento empático no estudante (talvez já existente, mas latente) à criança na condição de exclusão social ou situação de risco.

A forma de viabilizar essa prática foi estabelecer parceria com uma organização não-governamental, o Centro Comunitário da Criança e do Adolescente (CGCA), entidade do terceiro setor gerida pela Arquidiocese de São Paulo, cuja missão consiste em promover a construção da cidadania e o desenvolvimento integral de crianças e adolescentes em situação de risco pessoal e social.

O projeto de extensão em pauta, existente há dez anos, está vinculado às disciplinas ENP38I - Enfermagem na saúde da criança e ENPI75 - A criança em situação de violência: subsídios para a atuação do profissional de saúde, do Departamento Enfermagem Materno-Infantil e Psiquiátrica (ENP), que tem firmado convênio de estágio com o CGCA desde 2002. 
Desse modo, o desenvolvimento de atividades teórico-práticas de enfermagem no CCGA vem possibilitando aos estudantes a oportunidade de apreender a realidade da criança em situação de exclusão para organizar o serviço de saúde e a assistência de enfermagem condizente com sua condição de existência. Nesta perspectiva, o atendimento de saúde em questão tem o objetivo de levar o aluno a identificar as necessidades peculiares da criança em situação de exclusão social, e propor e executar cuidados de enfermagem compatível com as necessidades identificadas.

\section{MATERIAIS E MÉTODO}

Além dos estágios das disciplinas de graduação referidas, o atendimento às crianças do CGCA desenvolve projetos das Pró-Reitorias de Cultura e Extensão e de Pesquisa da USP e da Pós-Graduação da Escola de Enfermagem.

\section{PARTICIPANTES}

Já participaram do projeto, entre 2000 e 2009 , aproximadamente, IOO alunos de graduação da disciplina ENP38I; 40 alunos de graduação da disciplina ENP I75; dois alunos de graduação da Bolsa Trabalho da Coordenadoria de Assistência Social (Coseas); um aluno de graduação da Bolsa Aprender com Cultura e Extensão; dois alunos de pós-graduação do Programa de Aperfeiçoamento de Ensino (PAE); e dois alunos, um de graduação e outro de pós-graduação, do projeto de Fomento às Iniciativas de Cultura e Extensão da PRCEU.

\section{PROJETOS DE PESQUISA VINCULADOS}

As pesquisas vinculadas ao CGCA foram "Desenvolvimento da sexualidade da criança em situação de risco" (mestrado); "Cuidado de enfermagem à criança em situação de exclusão social na ótica de estudantes de enfermagem" (iniciação científica do projeto Ensinar com Pesquisa da Pró Reitoria de Pesquisa); e "Vivências e percepções de adolescentes em situação de exclusão social sobre a violência" (mestrado).

\section{EMPRESAS PARCEIRAS}

O projeto conta com a parceria de uma empresa privada (serviço ambulatorial da SAMCIL Planos de Saúde), de uma ONG (serviço de oftalmologia da Associação Budista e Beneficente Assistência Social Tzu-
Chi) e de uma universidade (serviço de psicologia da Pontifícia Universidade Católica de São Paulo).

\section{INSTRUMENTOS UTILIZADOS NAS CONSULTAS:}

Os sete primeiros instrumentos abaixo relacionados foram elaborados pelos alunos (em conjunto com a docente responsável) e são atualizados semestralmente. São eles:

- Formulário (roteiro) de avaliação da saúde da criança. (Anexo)

- Manual de avaliação física e comportamental da criança.

- Termo de consentimento dos pais em que se autoriza a avaliação da criança.

- Ficha de encaminhamento para atendimento de saúde nos serviços parceiros: ambulatório da $\mathrm{Sa}-$ mcil, psicolologia/PUC e Unidade Básica de Saúde (UBS) regional.

- Ficha de encaminhamento para serviço oftálmico da Tzu-Chi.

- Solicitação de presença dos pais/responsáveis para entrevista.

- Declaração de presença dos pais/responsáveis no GCCA.

- Gráficos de desenvolvimento pondero-estatural de meninas e meninos do Ministério da Saúde e da Organização Mundial de Saúde, para cálculo de percentis de peso e altura.

- Escala Optométrica de Snellen, utilizada para verificação da acuidade visual.

- Livro: ALEXANDER, M. M.; BROWN, M. S. Diagnóstico na Enfermagem Pediátrica. Andrei, 1978.

- Materiais de verificação do desenvolvimento cognitivo.

- Instrumentos de avaliação física (estetoscópio, esfigmomanômetro, balança, termômetro, luvas, fita métrica, otoscópio e materiais descartáveis).

\section{RESULTADOS}

As atividades desenvolvidas por meio da avaliação física e do desenvolvimento da criança, das entrevistas e da acuidade visual possibilitaram identificar alguns problemas de saúde que são encaminhados aos serviços parceiros do CGCA.

A partir de 2005, os alunos passaram a elaborar relatórios anuais dos atendimentos, contendo todas as 
atividades realizadas, o número de crianças atendidas e os problemas identificados. O número de crianças avaliadas depende do número de alunos disponíveis e das estratégias de ensino programadas a cada ano.

Nas avaliações das crianças, são identificados problemas de ordem física e psicossocial, além de situações de vulnerabilidade à violência. Foram examinadas: 3I crianças em 2009; 43 em 2008; 2 I em 2007; 108 em 2006 e 52 em 2005. Em 2007, 68 crianças foram avaliadas somente em relação à acuidade visual. Os gráficos abaixo expressam os números de problemas identificados nos anos especificados. Os problemas mais comuns foram odontológicos e de acuidade visual devido à dificuldade de acesso a esse tipo de serviço de saúde. A higiene precária revelava a negligência dos cuidados paternos.

\section{DADOS IDENTIFICADOS}

\section{GRÁFICO 1}

Problemas Físicos 2009

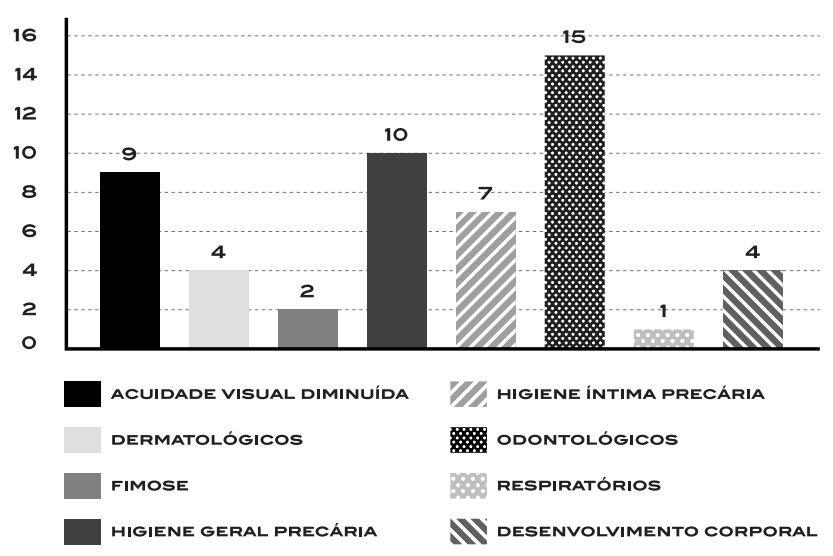

\section{GRÁFICO 2}

Problemas Físicos 2008

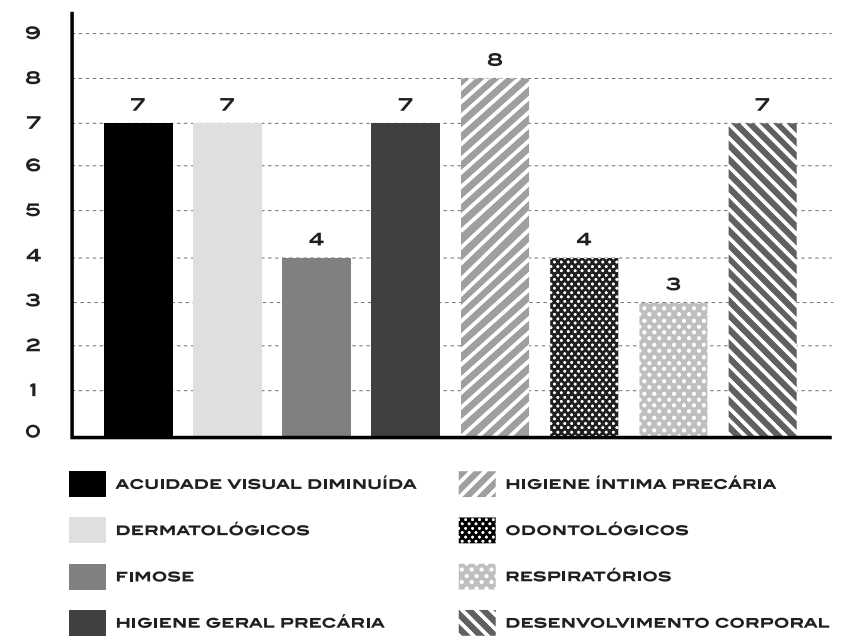

GRÁFICO 3

Problemas Físicos 2007

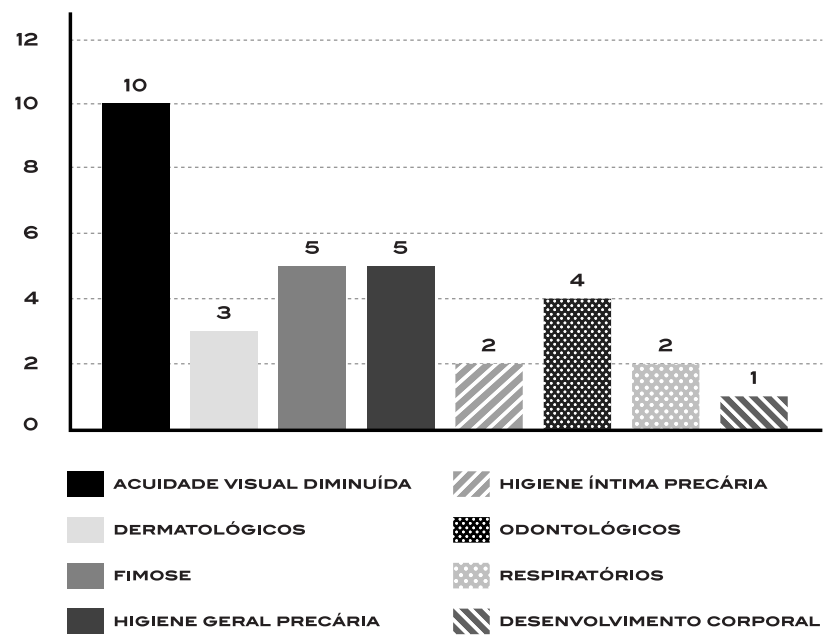




\section{GRÁFICO 4}

Problemas Psicossociais 2007

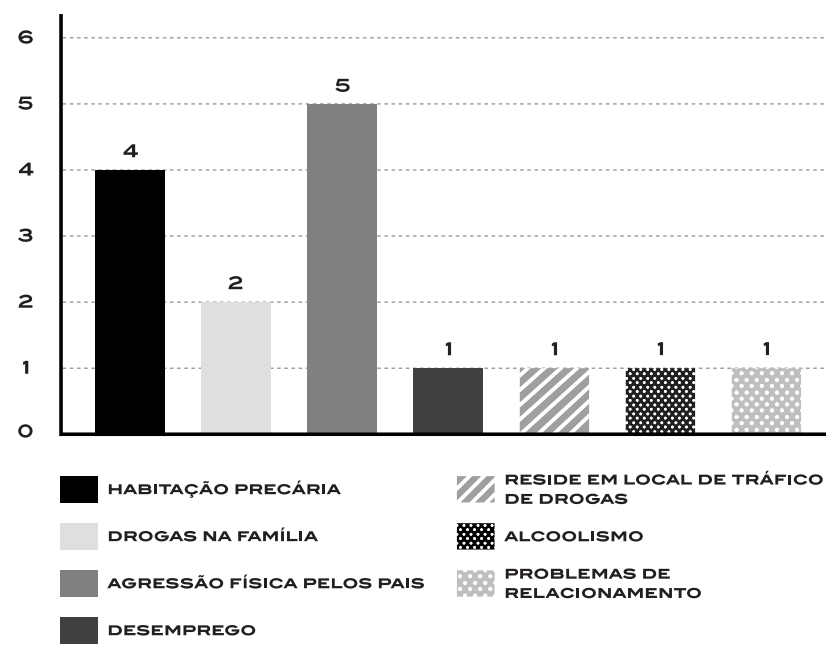

\section{GRÁFICO 5}

Problemas Físicos 2006

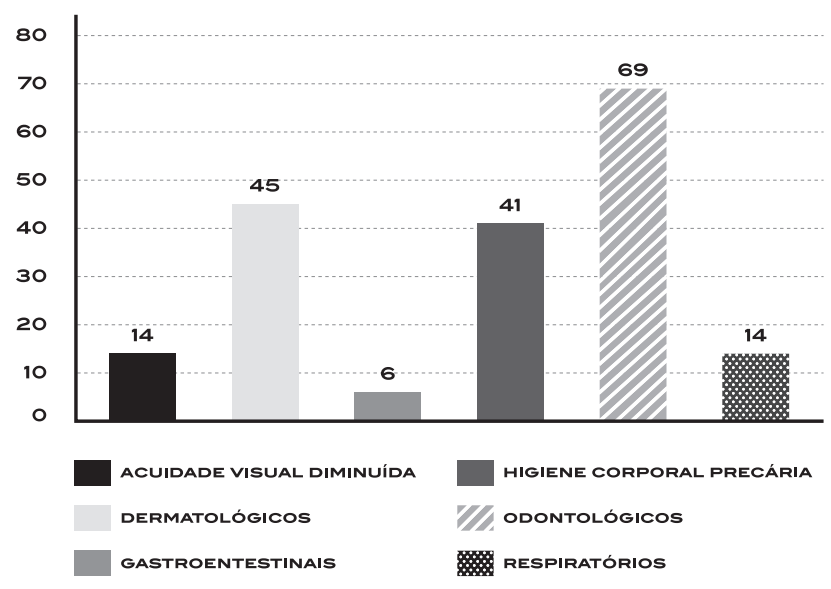

\section{GRÁFICO 6}

Problemas Psicossociais 2006

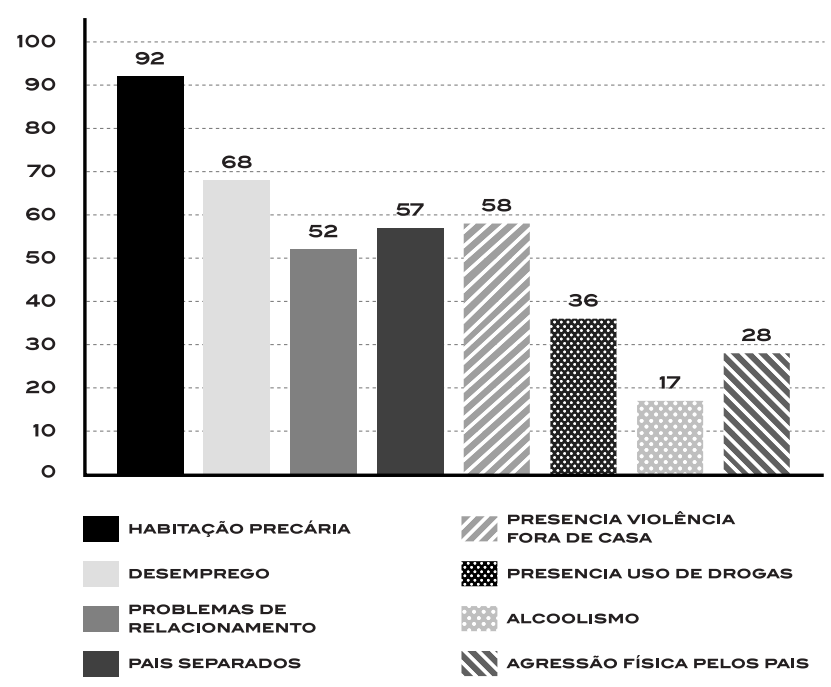

\section{GRÁFICO 7}

Problemas Físicos 2005

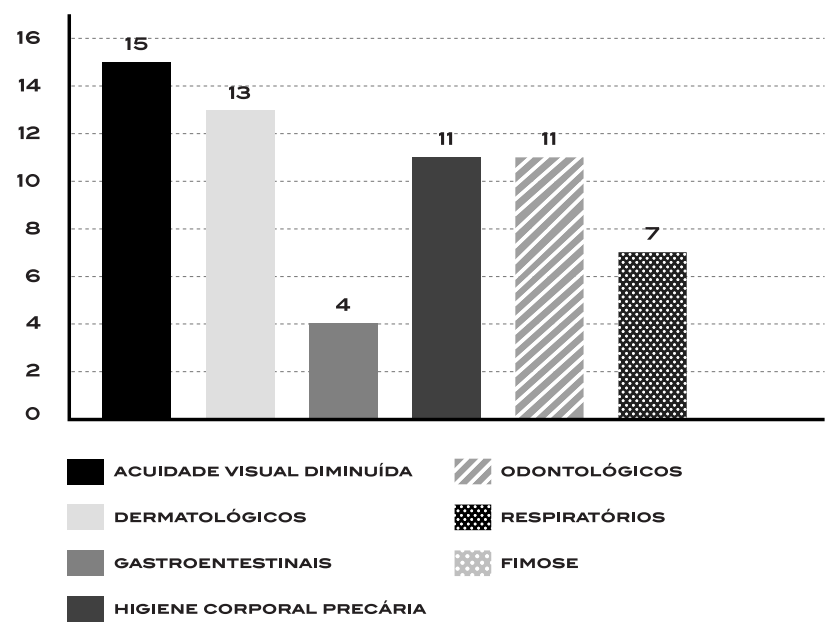




\section{GRÁFICO 8}

Problemas Psicossociais 2005

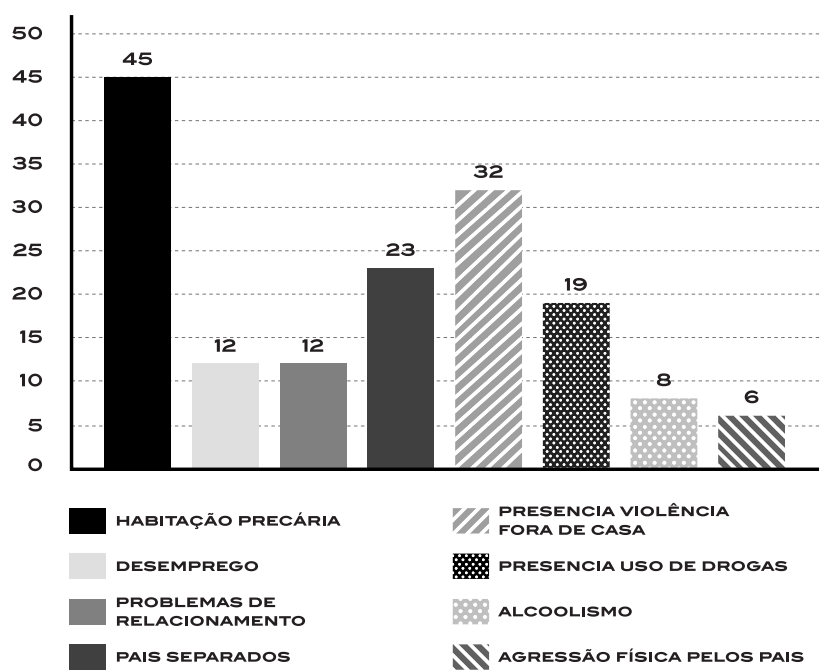

\section{DESENVOLVIMENTO DAS ATIVIDADES}

Os estudantes executaram avaliação física do crescimento e do desenvolvimento das crianças; coleta de informações sobre a história pessoal e familiar das crianças e suas condições habitacionais; e exame de acuidade visual. Estabeleceram prioridades de intervenção de enfermagem que consistiram em: cuidados imediatos (assepsias, hidratação oral, controle de febre); controle de vacinação; orientações individuais e em grupo (sobre higiene corporal, exercícios de retração do prepúcio); comunicação terapêutica; ações educativas (oficinas sobre higiene bucal, saúde/ doença, direitos da criança e questões sexuais); seleção de crianças para encaminhamento aos serviços de saúde parceiros; evolução de enfermagem (reavaliação dos problemas identificados nas avaliações anteriores); orientação aos familiares (sobre serviços públicos acessíveis; pediculose e higiene pessoal, violência doméstica); visita domiciliar; parecer sobre suspeita de violência doméstica contra a criança; elaboração e aperfeiçoamento dos formulários de atendimento.

Os dados obtidos foram registrados em formulários individuais, os quais compõem os prontuários das crianças e são arquivados na sala de enfermagem. Os dados coletados permitiram a realização de um levantamento dos problemas físicos e psicossociais das crianças e possibilitaram a elaboração de prescrições de enfermagem. Nestas, registraram-se as dúvidas e queixas das crianças durante o atendimento para organizar as intervenções de enfermagem e os encaminhamentos ao serviço ambulatorial de pediatria e dermatologia da Samcil (por intermédio da coordenação do (CGA) e à Associação Budista de Beneficência e Assistência Social Tzu Chi ${ }^{\dagger}$.

Os casos em que as crianças apresentaram algum indício (comportamental, físico ou discursivo) suspeito de serem vítimas de qualquer forma de violência, foram notificados à coordenação do CCCA.

Uma das pesquisas de mestrado consistiu em retratar e analisar a visão da criança em situação de risco pessoal e social em relação à sua sexualidade: concepções formadas, experiências, sentimentos gerados e efeitos sobre seu desenvolvimento. A outra dissertação consistiu em apreender a representação social do adolescente em situação de exclusão social sobre a violência em seu cotidiano. A pesquisa de iniciação científica, por sua vez, teve a finalidade de identificar quais são os cuidados que os alunos de graduação julgam importantes para a criança em situação de exclusão social.

\section{DISCUSSÃO}

$\mathrm{O}$ atendimento dirigido à população de risco é uma área emergente de atuação da saúde devido ao elevado índice de morbidade e mortalidade infantil decorrentes do estado de vulnerabilidade da criança socialmente excluída. Essa situação vem desencadeando algumas ações em âmbito técnico e acadêmico.

$\mathrm{O}$ atendimento de saúde no GCGA buscou inserir o estudante de enfermagem com o intuito de capacitálos a planejar, intervir e avaliar o atendimento prestado. Além de proporcionar oportunidade de aprendizagem aos alunos, o projeto tem beneficiado as crianças com o atendimento de saúde, a obtenção de óculos, tratamentos clínicos e oficinas educativas para o autocuidado, além de possibilitar o acesso à investigação científica que tende a gerar a intervenção prática imediata.

\footnotetext{
$\dagger \quad$ A ONG forneceu exames oftálmicos e óculos em 2007 e 2008 para crianças que tiveram baixa acuidade visual que foi medida com a carta de Snellen e algumas delas obtiveram lentes corretivas.
} 
O projeto iniciou com recursos físicos e materiais improvisados. Dependia de materiais doados pelos participantes (de ambas as instituições, das alunas e da professora) para a realização das atividades, mas a riqueza de experiências que o campo oferecia aos alunos compensava suas limitações. Aos poucos, o diretor do GGCA, sempre muito empenhado em melhorar a condições das crianças, forneceu as condições necessárias para o desenvolvimento das atividades.

A dificuldade maior consistia em obter um serviço ágil e próximo ao CCCA para dar prosseguimento ao atendimento dos problemas identificados pelos alunos. Estes detectavam problemas de saúde nas crianças, mas era necessário conquistar serviços parceiros para atender aos casos que demandavam atenção de profissionais especializados. A parceria com a Samcil resolveu grande parte do problema, mas não todos.

Uma das incumbências dos alunos consiste em buscar parcerias que atendam demandas, tais como a necessidade de óculos e o tratamento dentário para algumas crianças. Por falta de recursos humanos, o consultório odontológico que a entidade possui ficou inativo por alguns anos, mas a direção do CGCA conseguiu reativá-lo. Além disso, a iniciativa de uma aluna gerou o atendimento da ONG Tzu Chi.

A evolução do trabalho mostrou que o projeto é viável porque demanda baixo custo. À medida que se consolidava, ia conquistando recursos humanos por meio de bolsistas e, em 2008, obteve apoio financeiro da Pró-Reitoria de Gultura e Extensão Universitária da USP (PRGEU) para obtenção de materiais de consumo.

Apesar de sua evolução, o projeto enfrenta sérias dificuldades. Uma delas consiste no fato de ser sustentado por apenas um docente. Outra dificuldade surgiu recentemente com a mudança curricular da Escola de Enfermagem, pois atualmente prioriza outros projetos em andamento, o que levou à redução do número de estudantes disponíveis para o atendimento no GCGA. Contudo, o impacto da mudança curricular sobre o projeto não desestimula a docente responsável porque o presente projeto surgiu na adversidade e será nela que continuará prosperando.

\section{CONCLUSÃO}

O projeto tem potencial para ser replicado e, inclusive, foi desenvolvido também para mostrar que é viável e desejável que seja replicado em outras ONGs em parcerias com universidades e a iniciativa privada. Alguns ex-alunos manifestaram desejo de programar, em seu local de trabalho, atendimento de saúde que envolva profissionais e estudantes de saúde com a participação de empresas e universidades. $O$ projeto pode ser extensivo também a saúde da mulher, do idoso e de demais grupos sociais. Para alcançar essa meta, é necessário divulgar a proposta e organizar cursos de difusão para orientar sobre o processo de implementação e os requisitos necessários.

\section{AGRADECIMENTOS}

Às Pró-Reitorias de Cultura e Extensão Universitária e de Pesquisa da USP pelo apoio financeiro ao projeto e aos bolsistas.

\section{REFERÊNCIAS BIBLIOGRÁFICAS}

[1] ORGANIZAÇÃO MUNDIAL DE SAÚDE. Classificação estatística internacional de doenças e problemas relacionados à saúde: Classificação Internacional de Doenças. São Paulo: Edusp, I995.

[2] RIBEIRO, M. O. A saúde da família em situação de exclusão social. In: BRASIL. Manual de Enfermagem/Instituto para o Desenvolvimento da Saúde. Universidade de São Paulo. Ministério da Saúde. Brasília: Ministério da Saúde, 2001.

[3] SILVA, H. O; SILVA, J. S. Análise da violência contra a criança e o adolescente segundo o ciclo de vida no Brasil: conceitos, dados e proposições. São Paulo: Global/UNICEF, 2005. 